\title{
The electromagnetic field simulation and analysis of curved radiator
}

\author{
Lizhi Jiang ${ }^{1, a}$ \\ ${ }^{1}$ No.3 Renmin East Road,Kunming,Yunnan,650051,China. \\ akmjlz@163.com
}

Keywords: curved radiator, electromagnetic, polarity

\begin{abstract}
With the development of electromagnetic simulation technology, demand for electromagnetic radiation sensor is improving. The reasonable design of transmitter electromagnetic simulation technology has become a key. In this paper, the curved radiator of the electromagnetic radiation field by finite element simulation, and carries on the analysis and comparison for different incentives, lay the foundation for engineering application.
\end{abstract}

\section{Introduction}

With the development of electromagnetic simulation technology, radiator shape development from rules to the irregular, from bulk to miniaturization. Radiator design directly affect electromagnetic simulation results, rational design of radiator is particularly important, and become a widespread concern in the field of research direction.Most equations of electromagnetic field theory based on relatively rules bar radiator for evolution and computative, if using arc and other special-shaped radiator its will be very big amount of calculation, and prone to error. This paper with the help of eddy current field analysis functions of Ansys software Maxwell, single and two curved radiator space electromagnetic radiation field were analyzed, and the optimization design and incentives to make the space radiation field with a high energy level, to provide technical support for engineering application.

\section{Maxwell software profile}

Ansys software Maxwell is an international popular large general finite software package, is a powerful electromagnetic field simulation tool, mainly used in electric field, magnetic field, eddy current field, thermal field in areas for the calculation and analysis. It is based on maxwell differential equations, finite element discrete form, the changes of electromagnetic field calculation in the engineering for the large matrix, in the premise of guarantee the calculation accuracy and fast speed, provide consistent with the Windows operating habits and friendly user interface, intuitive, and convenient to use. Have smart grid subdivision function, can be manually subdivision, and automatic subdivision mesh save tedious work. A powerful model of 2D and 3D model simulation analysis pattern, according to the symmetry and the complexity of the design model to choose the appropriate analysis pattern, in order to simplify the analysis data. In addition also has the advantages of distributed computing and parallel computing to calm in the face of growing simulation model. They are for a single electromagnetic numerical calculation, also can conduct joint simulation of the whole system.

\section{Curved radiator modeling and analysis}

In theory to solve the curved radiator the size of the magnetic moment and phase is very difficult and tedious, it depends on the structure size, installation of the coil and core material of magnetic properties, the working frequency and the size of the coil through the current. In order to be more intuitive and visualization analysis arc magnetic field distribution of the radiator, this article uses the Ansys company MAXWELL software detailed analysis of its spatial distribution field. 
Are discussed below under the condition of double curved radiator symmetrical distribution, under the condition of with reverse phase current respectively with incentives to its space magnetic field distribution, and comparative analysis.

Two curved radiator model is set up as shown in figure 1 , the two curved radiator based on the $\mathrm{XY}$ plane, in XZ plane symmetric distribution and leave a gap in the middle. The simulation set specific parameters are as follows:

1. Core materials: iron;

2. Core size: inner diameter $160 \mathrm{~mm}, 170 \mathrm{~mm}$ diameter;

3. Enameled wire: thickness $5 \mathrm{~mm}$;

4. Number of ampere turns: 3223 (current and the product of the number of turns);

5. Frequency: $600 \mathrm{~Hz}$.

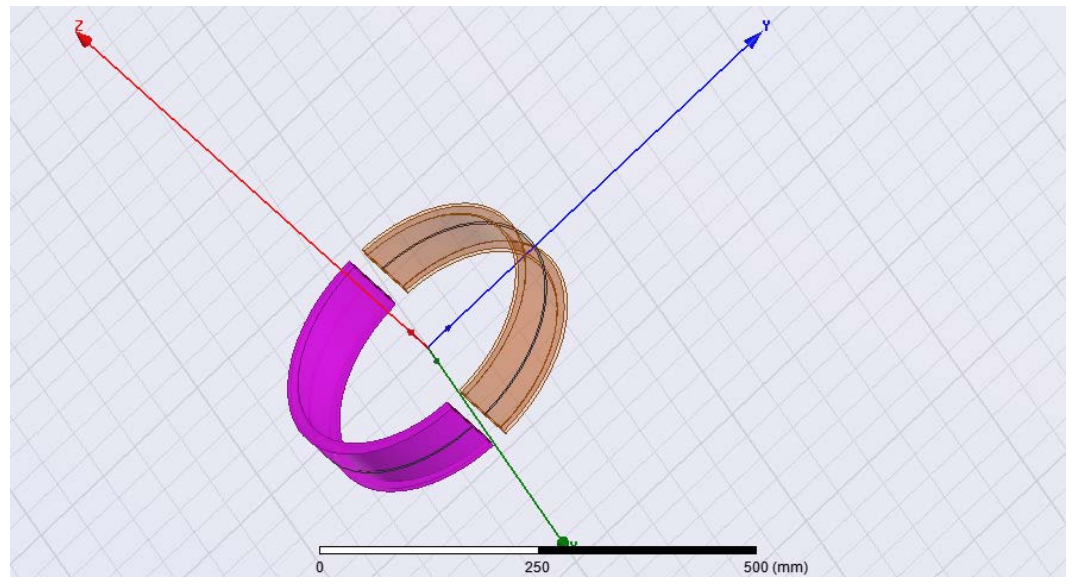

Figure 1 Radiator model diagram

In order to give full consideration to model the space magnetic field distribution, to set up two kinds of incentives, the first for the same polarity incentives (that is, the adjacent two extremely radiator with polarity), the second for different polarity stimulation. Analysis of the distribution of magnetic field by means of the simulation analysis in detail

First analyze the first case with polarity incentives, the manner of its relatively the same two radiator poles. By figure 2 planar magnetic field distribution can be seen that two symmetrical distribution of radiator always are in symmetrical distribution of the magnetic induction intensity on the plane. Present as a class in the XY plane circular field distribution, better symmetry, symmetry center energy is strongest. In the XZ plane and are distributed in the YZ plane class figure 8, the center of minimum energy of the symmetric line, two levels of energy is strongest.

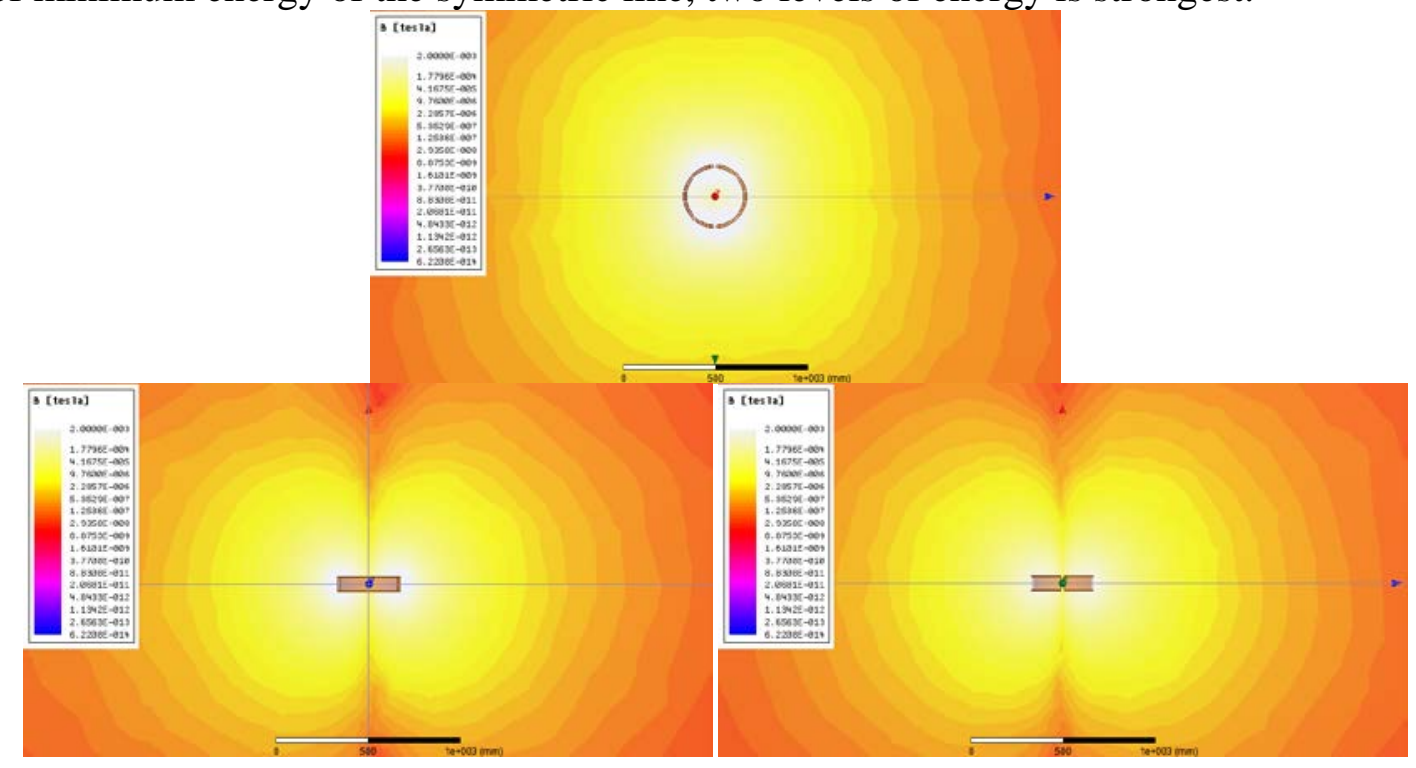

Figure 2 surface magnetic field distribution (up: XY plane, lower left: XZ plane, lower right: YZ plane) 
Figure 3 for curved radiator in the same polarity incentive mode of space electromagnetic induction intensity distribution. Four image representing the top view and left view, front view of the magnetic field and PLD figure. Comprehensive analysis of the four pair of electromagnetic field induction intensity distribution, found that curved radiator magnetic field in the same polarity incentive mode distribution is symmetry, and the $\mathrm{YZ}$ plane assumes the circular distribution of magnetic field. This suggests that the incentive mode of magnetic induction intensity distribution has stronger symmetry, and the axial distribution. Also because of its magnetic field is symmetrical distribution, is very beneficial for electromagnetic simulation technology.
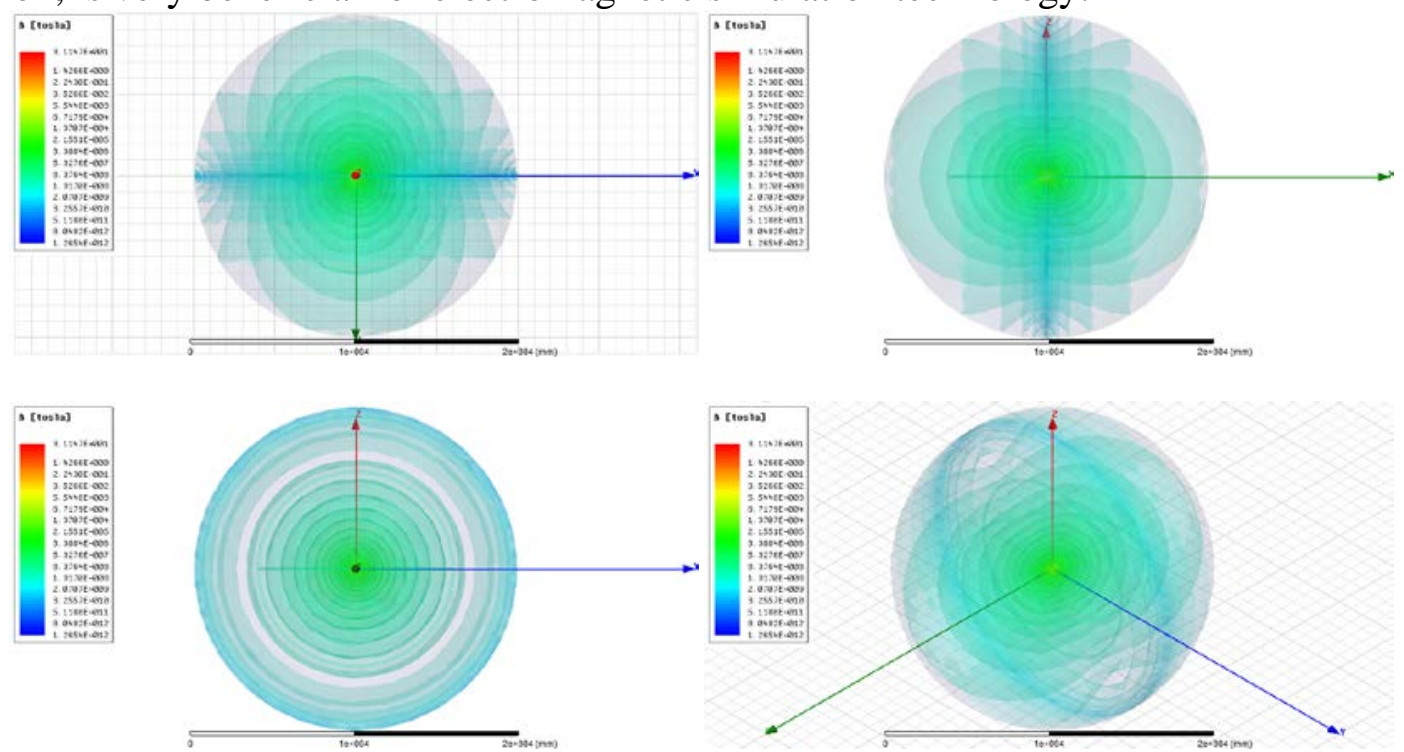

Figure 3 Space electromagnetic radiation field distribution diagram (upper left: top view, upper right: left view, bottom left: front view, lower right: 3D distribution map)

Figure 4 shows the coordinate origin as the center, followed by in XY (MAG_B), YZ (MAG_B_1), ZX (MAG_B_2) three planes within a radius of 6 meters in circumference of the magnetic induction intensity curve. XY (Mag_B), ZX (Mag_B_2)two plane on the circumference of a circle of magnetic induction intensity is sine curve, the YZ plane (Mag_B_1) constant energy on the circumference of a circle, it shows that the symmetry plane energy distribution.

The analysis of second kinds of heteropolarity incentive methods, the manner of its relative two radiator pole. By figure 5 planar magnetic field distribution can be seen that two symmetrical distribution of radiator always are in symmetrical distribution of the magnetic induction intensity on the plane. In the XY and XZ plane its present for elliptic far field distribution, better symmetry, symmetry center energy is strongest. In the YZ plane assumes the circular distribution, symmetry is best, center of symmetry energy is strongest.

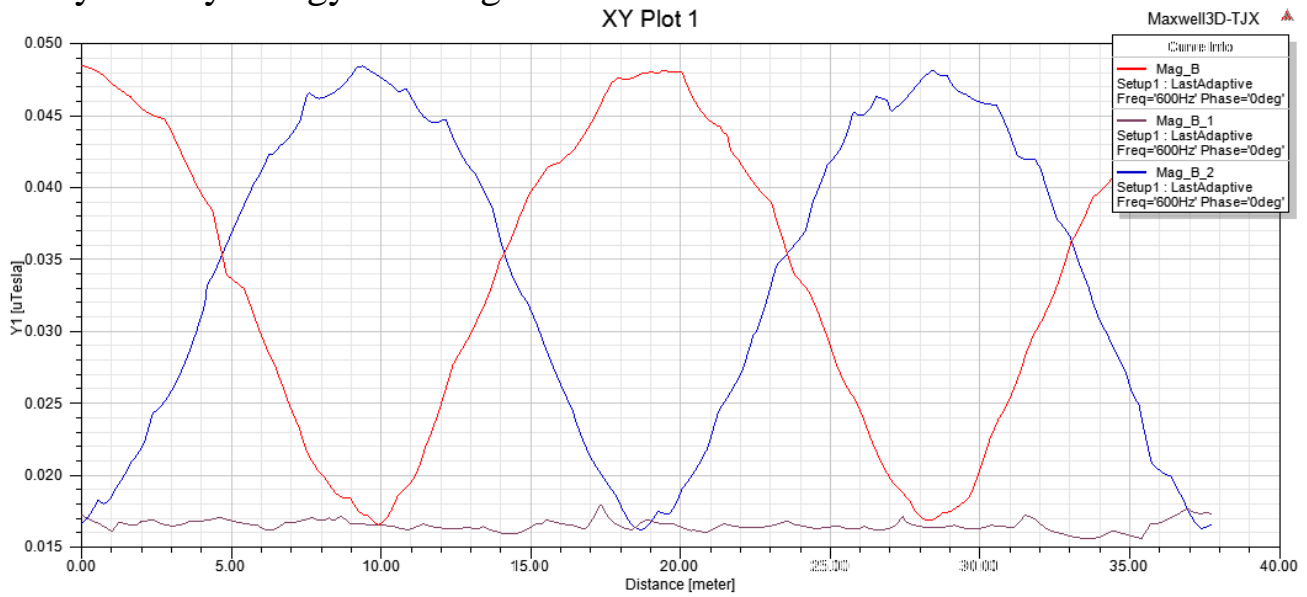

Figure 4 Magnetic induction intensity distribution on the circumference of 6 meters 
Figure 6 for curved radiator under different polarity incentives of space electromagnetic induction intensity distribution. Comprehensive analysis of the four pair of electromagnetic field induction intensity distribution, it can be seen that the curved radiator magnetic field under the different polarity incentives have a certain symmetry but not completely symmetrical, general poles slightly deflection phenomenon. Figure 7 planar circular magnetic induction intensity distribution analysis, different polarity incentives magnetic induction intensity of symmetry is not like the polarity, but the magnetic induction intensity fluctuation is less than the same polarity incentives.

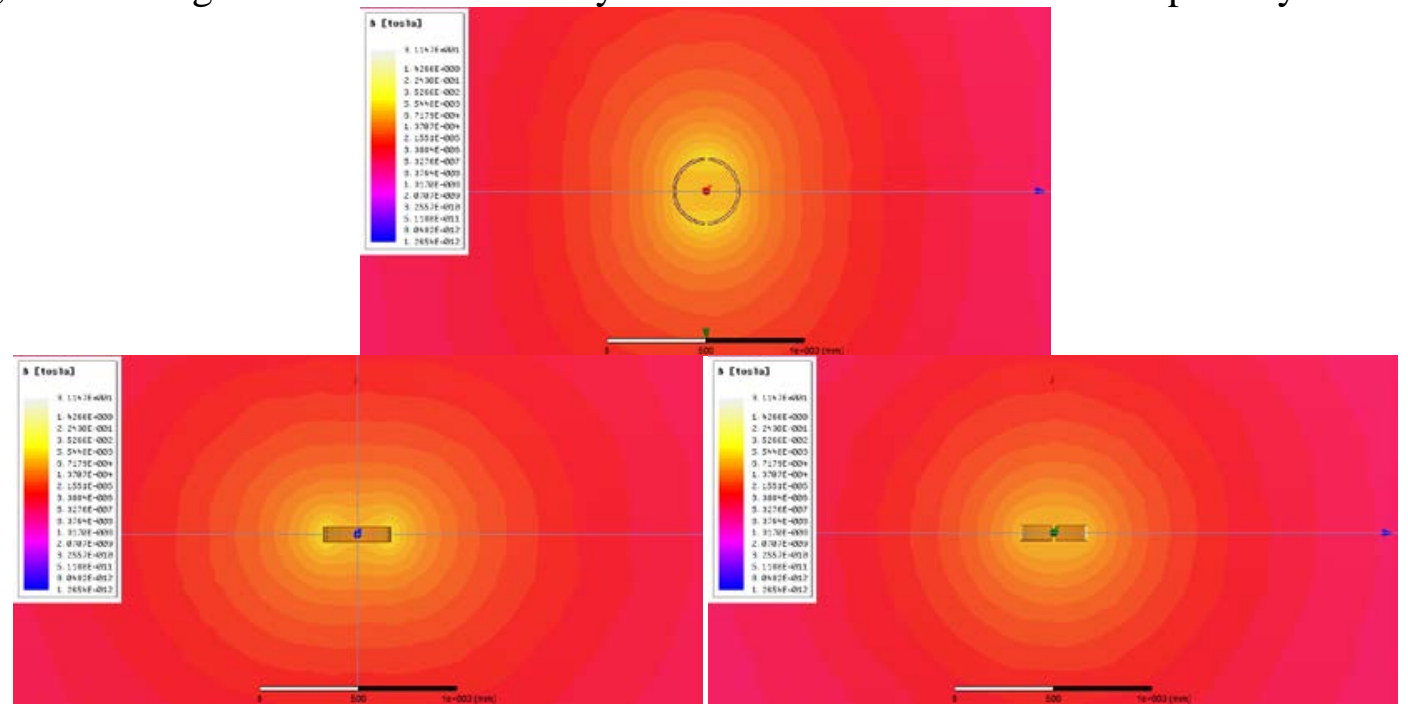

Figure 5 surface magnetic field distribution (up: XY plane, lower left: XZ plane, lower right: YZ plane)

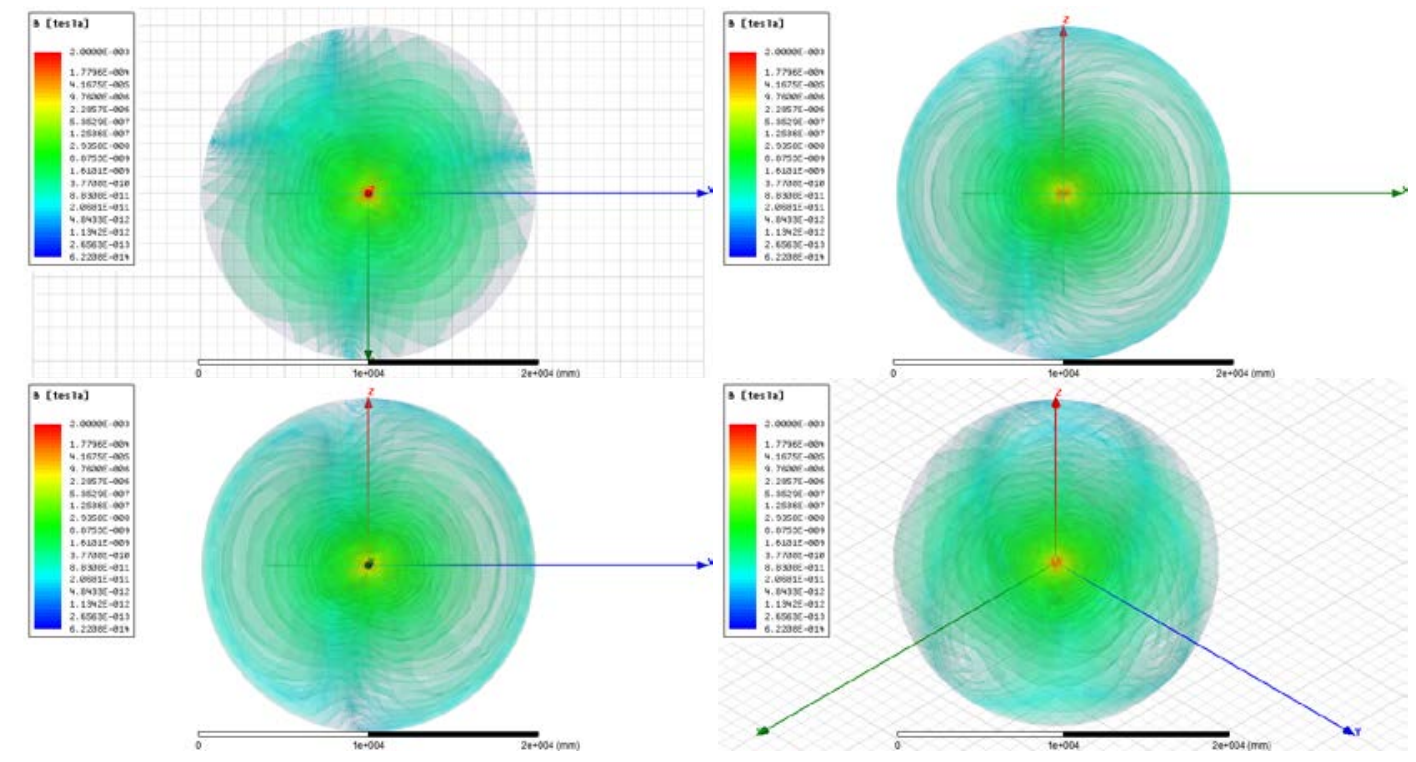

Figure 6 space electromagnetic radiation field distribution diagram (upper left: top view, upper right: left view, bottom left: front view, lower right: 3D distribution map) 


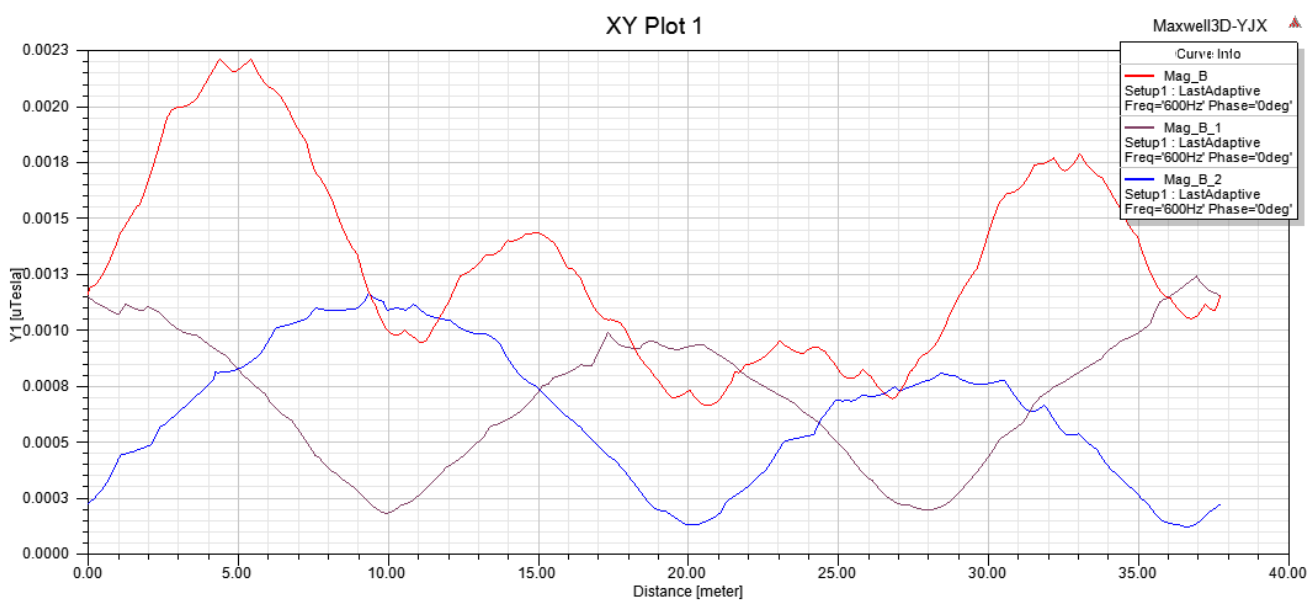

Figure 7 Magnetic induction intensity distribution on the circumference of 6 meters

\section{Conclusion}

This paper makes analysis and comparison on the magnetic field distribution of arc radiator in two kinds of incentive mode. Homopolar incentive the electromagnetic field distribution as well as symmetrical, spherical magnetic induction intensity in addition to the YZ plane, other planes were energy fluctuation and different incentive mode of 10 times above, the need for good symmetry environment use. Varied polarity incentive mode of the electromagnetic field distribution of symmetry as the polarity, but spherical magnetic induction intensity fluctuation is less than that of the same polarity. According to the actual demand of space electromagnetic field distribution and the intensity fluctuation of the magnetic field, the appropriate excitation method is chosen.

\section{References}

[1] Zhao Bo, Zhang Hongliang: Application of Ansoft 12 in engineering electromagnetic field, China WaterPower Press

[2] ANSYS Maxwell 3D Field Simulator v15 User's Guide, ANSYS Inc.

[3] ANSYS Maxwell Field Simulator v15 - Electrical Machine Design Reference, ANSYS Inc.

[4] Ansoft Maxwell Official Guide 1994-2000, Ansoft Corporation.

[5] ANSYS Maxwell 3D Field Simulator v16 User's Guide, ANSYS Inc. 